Pacific Journal of Mathematics

ABSTRACT RIEMANNIAN STRATIFICATION 


\title{
ABSTRACT RIEMANNIAN STRATIFICATIONS
}

\section{Giacomo Monti BRagadin}

\begin{abstract}
Let $A$ be an abstract stratification. Assume that every stratum $X$ is a riemannian manifold. We first give some conditions, under which it is possible to endow $A$ with a suitable metric extending a well known technique of riemannian manifolds. Then stronger conditions are introduced in order that the metric space $A$ becomes a $G$-space. An abstract stratification with all the above conditions is called an abstract riemannian stratification. Whitney stratifications are, in a natural way, riemannian.
\end{abstract}

Introduction. An abstract stratification is the disjoint union of a locally finite set of smooth manifolds, the strata, glued together according to certain rules (see [9], [13], [15], [16]).

The basic tools of differential calculus, smooth functions, vector fields and differential forms, can be also defined for an abstract stratification by means of "controlled" collections of "tools". Classical results can be extended to abstract stratifications (e.g. de Rham's theorems, see [15], [8]) in this way.

On the other hand a controlled approach seems to be useless when we take into account a riemannian structure: control conditions on the metric of the strata are too strict (bundle-like metrics, see [11], are not controlled in general).

The purpose of this paper is to obtain weaker conditions in order that riemannian metrics of the strata glue together in an appropriate manner. In $\S 1$ sufficient conditions are given for extending the fundamental theorem of the riemannian geometry; in $\S 2$ a definition of compatibility among the riemannian structures of the strata is given and connections with the structure of $G$-space are studied. It is shown that the axioms of finite compactness, convexity, local prolongability are satisfied in mild hypotheses, whereas local uniqueness and existence of geodesic coordinates must be required (the former is an open question even in manifolds with boundary, see [1]).

0. Notation. 1. By a manifold we shall always mean a smooth manifold; topologically it is a connected, paracompact space. For any 
manifold $M$ let $\mathscr{C}^{\infty}(M)$ be the ring of smooth real valued functions on $M$, and let $T_{p} M$ be the tangent space at $p \in M$.

If $g$ is a riemannian metric on $M$, for each $v \in T_{p} M$ the length $\|v\|=g_{p}(v, v)$ is defined; if $g_{i j}$ are the components of $g$ with respect to local coordinates centered at $p$ and $v^{i}$ are those of $v$, then

$$
\|v\|=\left(\sum g_{i j}(p) v^{i} v^{j}\right)^{1 / 2}
$$

The length of a differentiable curve $L$ parametrized by $\varphi:[a, b] \rightarrow$ $M$ is the integral of its velocity vector:

$$
|L|=\int_{a}^{b}\left\|\varphi_{t}\right\| d t
$$

An abstract stratification is a triple $(A, \mathscr{S}, \mathscr{T})$ where

- $A$ is a connected, locally compact topological space with a countable base;

- $\mathscr{S}$ is a locally finite partition of $A$ into locally closed subsets, the strata, such that if $X, Y \in \mathscr{S}$ and $X \cap \bar{Y} \neq \varnothing$, then $X \subset \bar{Y}$; moreover every $X \in \mathscr{S}$ is a manifold without boundary with the induced topology;

- $\mathscr{T}=\left\{T_{X}, \pi_{X}, \rho_{X}\right\}$ is the family of the control data:

$-T_{X}$ is an open neighbourhood of $X$ in $A$, $-\pi_{X}: T_{X} \rightarrow X$ is a continuous retraction, $-\rho_{X}: T_{X} \rightarrow[0,+\infty)$ is the tubular function (see [9], [13]).

We shall often denote the triple $(A, \mathscr{S}, \mathscr{T})$ by $A$ for short.

For each integer $r \geq 0$ define $\mathscr{S}^{r}=\{X \in \mathscr{S} \mid \operatorname{dim} X \leq r\}, A^{r}=$ $\bigcup_{X \in \mathscr{S}^{r}} X$. Then it is easy to define, by restriction, control data $\mathscr{T}^{r}$ so that $A^{(r)}=\left\{A^{r}, \mathscr{S}^{r}, \mathscr{T}^{r}\right\}$ is an abstract stratification, called the $r$ skeleton of $A$. If $A=A^{(r)}$ for some $r \in \mathbf{N}$ we shall say that $A$ is finite dimensional and the smallest $r$ with this property is called $\operatorname{dim} A$. We shall always suppose that $A$ is finite dimensional; if $n=\operatorname{dim} A$ then $\sum A=A^{(n-1)}$ is non-empty and $A^{*}=A-\sum A$ is dense in $A$; each stratum $X$ is endowed with a riemannian metric $g_{X}$.

2. Let $(X, d)$ be a metric space.

We use the notation $(x y z)$ to indicate that $x, y, z$ are distinct points of $X$ and $d(x, y)+d(y, z)=d(x, z)$ (" $y$ lies between $x$ and $z$ ").

The length $\lambda(x)$ of a curve $x(t)(a \leq t \leq b)$ is defined in the usual way: for any partition $\delta: a=t_{0}<t_{1}<\cdots t_{k}=b$ we put $\lambda(x, \delta)=$ $\sum d\left(x\left(t_{i}\right), x\left(t_{i-1}\right)\right)$ and $\lambda(x)=\sup \lambda(x, \delta)$; the metric $d$ is intrinsic if $d(x, y)$ equals the infimum of the lengths of all curves from $x$ to $y$. 
A segment $T(y, z)$ is a curve from $y$ to $z$ of length $d(y, z)$ : it is a shortest join of $y$ to $z$ and any subarc is a segment.

A geodesic is a local isometry $\sigma: R \rightarrow X$; in particular a geodesic is locally a segment.

The metric space $(X, d)$ is called a $G$-space if

(i) the closed balls are compact;

(ii) the metric $d$ is intrinsic;

(iii) the axioms $P$ and $U$ (listed below) are satisfied.

Axiom $P$ (local prolongability): each point $p$ of $X$ has a neighbourhood $U_{p}$ such that for distinct $x, y$ in $U_{p}$ there exists $z \in X$ with $(x y z)$.

Axiom $U$ (uniqueness of the prolongation): let $i=1,2$. If $\left(x y z_{i}\right)$ holds and $d\left(z_{1}, x\right)=d\left(z_{2}, x\right)$, then $z_{1}=z_{2}$.

Condition (i) is often called finite compactness. Condition (ii) is equivalent (for finitely compact metric spaces) with the so-called Menger convexity: For each $x, y \in X(x \neq y)$ there exists a point $z \in X$ such that $(x y z)$. Conditions (i), (ii) ensure that segments exist, almost locally; if axiom $P$ holds then each segment can be extended to a geodesic, uniquely if axiom $U$ holds.

The most important results on $G$-spaces are contained in [5], [6], [7]. $G$-spaces include complete riemannian manifolds; the theory developed in [5], [6], [7] leads one to recognize which theorems pertain to $G$-spaces theory and which to riemannian geometry.

1. Riemannian metrics on abstract stratifications. Let $L$ be a curve parametrized by $\varphi:[a, b] \rightarrow A$. If im $(\varphi) \subset X$ for some stratum $X$ and $L$ is piece-wise differentiable (p.d.) curve of the manifold $X$, then $|L|$ is defined. If there exists a stratum $X$ such that $\operatorname{im}(\varphi) \subset \bar{X}$ and for $t \in[a, b)$ the restriction $L_{t}$ of $L$ to $[a, t]$ is p.d. in $X$, then we define $|L|=\lim _{t \rightarrow b}\left|L_{t}\right|$.

Finally if there exists a subdivision of $[a, b]: a=a_{0}<a_{1}<\cdots<$ $a_{n}=b$ such that the restriction $L_{i}$ of $L$ to $\left[a_{i}, a_{i+1}\right]$ has a length $\left|L_{i}\right|$ in the above sense, we put $\sum_{i=0}^{n-1}\left|L_{i}\right|=|L|$.

Definition 1. The curve $L$ is summable if $|L| \in \mathbf{R}$; for every pair $x, y$ of points of $A$ we define $d_{A}(x, y)=\inf \{|L| \mid L$ is summable with endpoints $x, y$ \}.

We shall prove that in suitable hypotheses $d_{A}$ is a metric on $A$ which 
induces the stratification topology. For this we need the following

DEFINITION 2. Let $A$ be an abstract stratification and a topological manifold. The $C^{0}$ structure is compatible with the smooth structures of the strata if for every coordinate system $(U, \varphi)$

(i) for each $x \in U$, if $X$ is the stratum which contains $x$, then there exists a neighbourhood $U_{x}$ of $x$ in $X$ such that $\left(U_{x}, \varphi \mid U_{x}\right)$ is a coordinate system of the smooth structure of $X$;

the $C^{0}$ structure is compatible with the riemannian structures of the strata if in addition:

(ii) let $\left\{U_{X, i}\right\}$ be an open covering of $X$ with coordinate neighbourhoods given by (i); then fix a refinement $\left\{V_{X ; i}\right\}$ and define

$$
\begin{aligned}
& m_{X, i}=\inf \left\{\sum_{1}^{\operatorname{dim} X}\left(g_{X, i}\right)_{h, k}(x) \alpha^{h} \alpha^{k} \mid \sum_{1}^{\operatorname{dim} X}\left(\alpha^{1}\right)^{2}=1, x \in V_{X, i}\right\}, \\
& M_{X, i}=\sup \left\{\sum_{1}^{\operatorname{dim} X}\left(g_{X, i}\right)_{h, k}(x) \alpha^{h} \alpha^{k} \mid \sum_{1}^{\operatorname{dim} X}\left(\alpha^{1}\right)^{2}=1, x \in V_{X, i}\right\} ;
\end{aligned}
$$

for every compact $K \subset A$ put

$$
m_{X, K}=\liminf _{\bar{V}_{X, i} \cap K \neq \varnothing} m_{X, i}, \quad M_{X, K}=\limsup _{\bar{V}_{X, i} \cap K \neq \varnothing} M_{X, i} .
$$

Then we require that for every $X \in \mathscr{S}$ and for every compact $K \subset A$

$$
0<m_{X, K}, \quad M_{X, K} \in \mathbf{R} .
$$

Theorem 1. Let $A$ be as in Definition 2. Then $d_{A}$ is a metric on $A$, which induces the underlying topology.

Proof. Let $p \in A$ and let $(U, \varphi)$ be a coordinate system centered at $p$. We can choose a number $\alpha>0$ such that $B_{\alpha}=D(0, \alpha) \subset \varphi(U)$. Then for every $r \in(0, \alpha]$ we put $K_{r}=\varphi^{-1}\left(B_{r}\right)$ and $K=K_{\alpha}$.

Now let $L$ be any summable curve contained in $K$, with endpoint $p$. Let $q$ be the startpoint of $L$ and let $x:[a, b] \rightarrow A$ be a parametrization of $L$. Let $\|L\|$ be the euclidean length of $\varphi^{0} L$.

We suppose at first that $p \in X$ and there exists a stratum $Y$ such that $X<Y$ and $L-\{p\} \subset Y$.

Then we can cover $L-\{p\}$ with coordinate neighbourhoods $Y_{Y, i}$ as in the Definition 2. If $\left\{V_{i}\right\}$ is the corresponding refinement, then there exist curves $L_{i}$ such that $L_{i} \subset V_{i}$ and $L_{i} \cap L_{i+1}$ is a point, for every $i$. 
If we put $\alpha_{i}=\left|L_{i}\right|, \beta_{i}=\left\|L_{i}\right\|, A_{n}=\sum \alpha_{i}, B_{n}=\sum \beta_{i}$ we get (see [4])

$$
\begin{aligned}
& \frac{A_{n}}{B_{n}}=\frac{1}{B_{n}} \sum \frac{\alpha_{i}}{\beta_{i}} \beta_{i} \leq \frac{1}{B_{n}} \sum M_{Y, i} \beta_{i} \leq \max _{1 \leq i \leq n} M_{Y, i}, \\
& \frac{A_{n}}{B_{n}}=\frac{1}{B_{n}} \sum \frac{\alpha_{i}}{\beta_{i}} \beta_{i} \geq \frac{1}{B_{n}} \sum m_{Y, i} \beta_{i} \geq \min _{1 \leq i \leq n} M_{Y, i},
\end{aligned}
$$

hence:

$$
0<m_{Y, K} \leq \frac{|L|}{\|L\|} \leq M_{Y, K} .
$$

The above (1) also holds in the general case. It is enough to give the proof for the case $L=L_{1} \cup L_{2}, L_{1} \cap L_{2}=\{q\}, L_{2}-\{q\} \subset Y$, $L_{1}-\{p\} \subset Z$ and $X<Z<Y$. It follows from (1) that

$$
m_{Y, K} \leq \frac{\left|L_{2}\right|}{\left\|L_{2}\right\|} \leq M_{Y, K} \quad \text { and } \quad m_{Z, K} \leq \frac{\left|L_{1}\right|}{\left\|L_{1}\right\|} \leq M_{Z, K} \text {, }
$$

hence

$$
\begin{aligned}
\frac{|L|}{\|L\|} & =\frac{\left|L_{1}\right|+\left|L_{2}\right|}{\left\|L_{1}\right\|+\left\|L_{2}\right\|} \\
& \leq \frac{\left\|L_{1}\right\| M_{Z, K}+\left\|L_{2}\right\| M_{Y, K}}{\left\|L_{1}\right\|+\left\|L_{2}\right\|} \leq \max \left(M_{Z, K}, M_{Y, K}\right), \\
\frac{|L|}{\|L\|} & \geq \min \left(m_{Z, K}, m_{Y, K}\right) .
\end{aligned}
$$

All together, if

$$
M=\max _{X \cap K \neq \varnothing} M_{X, K} \text { and } m=\min _{X \cap K \neq \varnothing} m_{X, K}
$$

one has for any summable curve $L$ contained in $K$

$$
0<m \leq \frac{|L|}{\|L\|} \leq M .
$$

For every $r \in(0, \alpha]$, if

$$
m_{r}=\min _{X \cap K_{r} \neq \varnothing} m_{X, K_{r}} \text { and } M_{r}=\max _{X \cap K_{r} \neq \varnothing} M_{X, K_{r}}
$$

one has $m=m_{a} \leq m_{r}$ and $M=M_{a} \geq M_{r}$, so that for every summable curve $L \subset K_{r}$ with $x(a)=q, x(b)=p$

$$
0<m\|\varphi(q)\| \leq m_{r}\|\varphi(q)\| \leq|L| \leq M_{r}\|L\|<M\|L\|
$$

where $\|\varphi(q)\|$ is the euclidean norm of $\varphi(q)$.

The theorem follows from (3) with a standard argument (see [2] or [4]). 
REMARKs. 1. The long spiral of $\mathbf{R}^{2}$ and more generally any euclidean curve with infinite length, equipped with the geodesic distance, does not satisfy Definition 2(ii).

2. In general $d_{A}$ does not induce on $X \in \mathscr{S}$ the metric $g_{X}$; nevertheless the immersion $X \rightarrow A$ is a local isometry if $X$ is maximal. In particular the geodesics of $X$ (with respect to $g_{X}$ ) are the traces on $X$ of those of $A$.

2. Compatibility. In the present section we shall consider only stratifications $A$ with the following properties:

$A$ is a topological manifold, the topological structure is compatible with the riemannian metrics $g_{X}, X \in \mathscr{S}$.

By Theorem 1 such a stratification is a metric space $\left(A, d_{A}\right)$; we are looking for condition which make this metric space a $G$-space. Finite compactness is the first axiom of $G$-spaces. So we are led to consider only stratifications compact in the sense of [13]I.D: each stratum $X$ is diffeomorphic with the interior of a manifold with boundary $V_{X}$ (see [13], [15] or [16]), which admits a complete riemannian metric, by the Hopf-Rinow theorem. It is easily seen that the resulting metrics on the strata satisfy the conditions of Definition 2.

The other axioms need some compatibility conditions among the metrics $g_{X}$.

The first requirement is that

$$
\lambda(L)=|L| \text { for each summable curve } L .
$$

If (4) holds, then

$$
d_{A}(x, y)=\inf \left|L_{x y}\right|=\inf \lambda\left(L_{x y}\right) \quad \text { for every } x, y \in A
$$

so that $d_{A}$ is intrinsic (see [7]). It follows that $\left(A, d_{A}\right)$ is convex (see [7] (1.5), also [6] (5.18)).

The second condition of compatibility is

for every $p \in A$ there exists a neighbourhood $U$ of $p$ such that if $\left\{x_{n}\right\},\left\{y_{n}\right\}$ are sequences in $U$ which con(5) verge respectively to some $x, y \in U$, then the segements $T\left(x_{n}, y_{n}\right)$ converge to the segment $T(x, y)$ in the Hausdorff metric (see [3] or [5]).

If (5) holds, it is sufficient to prove the axiom of local prolongability in the following situation:

$x, y$ belong to an open convex subset $U \subset A, x \in A^{*}, y \in \sum A$. 
For each $z \in U$, let $t_{z}$ be the first point of $T(x, z) \cap \sum A$. Put $T=\left\{t \in U \cap \sum A \mid \exists z \in A\right.$ such that (xtz) $\}$. We have to show that $T=U \cap \sum A$.

We shall divide the proof in two steps.

1st step: $T$ is closed in $U \cap \sum A$.

Let $\left\{t_{n}\right\}$ be a sequence of points of $T$ which converges to $t^{*} \in$ $U \cap \sum A$. For each $n \in \mathbf{N}$ let be $z_{n}$ such that $\left(x t_{n} z_{n}\right)$; because of the finite compactness of $A$, we can assume that $z^{*}=\lim z_{n} \in U$. Then by (5):

$T\left(t_{n}, z_{n}\right)$ converges to $T\left(t^{*}, z^{*}\right)$ and $T\left(x, z_{n}\right)$ to $T\left(x, z^{*}\right)$, so that it follows from $T\left(t_{n}, z_{n}\right) \subset T\left(x, z_{n}\right)$ that $T\left(t^{*}, z^{*}\right) \subset T\left(x, z^{*}\right)$. Hence $\left(x t^{*} z^{*}\right)$ holds and $t^{*} \in T$ as required.

2nd step. $T$ is dense in $U \cap \sum A$.

Otherwise there exists an open set $V \subset U \cap \sum A$ such that no segment with endpoint in $V$ has a prolongation. Let $a \in V$ be any point and let $\left\{a_{n}\right\}\left\{b_{n}\right\}$ be sequences in different components of $U-\sum A$ both converging to $a$. By $(5)$ the sequence $\left\{T\left(a_{n}, b_{n}\right)\right\}$ converges to $\{a\}$, so that there exists a sequence $\left\{v_{n}\right\}$, with $v_{n} \in T\left(a_{n}, b_{n}\right) \cap \sum A$ and $\lim v_{n}=v$. Then $v_{n} \in V$ for great $n$, a contradiction.

The question of finding sufficient conditions for the uniqueness of the prolongation is still open, even for manifolds with boundary (see [1]). We just observe that the following

$$
\lambda(L)=|L| \text { for every segment } L
$$

is necessary.

As a matter of fact, if $L$ is a segment and $\lambda(L) \neq|L|$ then $\lambda(L)<|L|$. It follows that there exist points $p_{1}, p_{2} \in L$ and a curve $L_{1}$ with endpoints $p_{1}, p_{2}$ such that $\left|L_{1}\right|<\left|L_{12}\right|$ (where $L_{12}$ is the restriction of $L)$. But $L_{12}$ is a segment, so the last inequality gives a contradiction.

A compact stratification $A$ with compatibility properties (4) and (5) and uniqueness of the prolongation is a $G$-space (as metric space $\left(A, d_{A}\right)$, see [6]).

Remark. Let $X, Y$ be strata of an abstract stratification $A$ with $Y \subset \bar{X}$ and $\operatorname{dim} X=\operatorname{dim} A$.

Let $g_{X}$ be a riemannian metric on $X$, such that: for every vector field $v$ on $Y$, if $\bar{v}$ is a controlled extension of $v$, then for every $y \in Y$, taken a sequence $\left\{x_{n}\right\}$ in $X$ converging to $y$, the sequence

$$
\left\|v_{n}\right\|=g_{X}\left(x_{n}\right)(\bar{v}, \bar{v})^{1 / 2}
$$


converges to a limit, which does not depend on the choice of $\left\{x_{n}\right\}$. By results of [5] it is possible to show that there exists a riemannian metric $g_{Y}$ on $Y$ such that $g_{Y}(y)(v, v)^{1 / 2}$ is equal to the limit above. In other words a $g_{X}$ as above induces a riemannian metric $g_{Y}$ for every stratum $Y \subset X$. If the strata $X \subset A$ with $\operatorname{dim} X=\operatorname{dim} A$ are equipped with a $g_{X}$ as above and for every $Y \subset \sum A$ the resulting $g_{Y}$ is the same (i.e. does not depend on the choice of $X$ such that $Y \subset \bar{X}$ ), the resulting collection $\left\{g_{Z}\right\}_{Z \in \mathscr{S}(A)}$ satisfies (4) and (5).

Definition 3. We shall call the collection $\left\{g_{Z}\right\}$ of the previous remark compatible and the abstract stratification riemannian.

Proposition. Let $A \subset \mathbf{R}^{N}$ be a Whitney stratification; equip every stratum $X$ with the induced riemannian metric. Then $A$ is a riemannian stratification.

COROLlARY 1. Every abstract stratification has a riemannian structure. (Use a realization, see $[\mathbf{1 0}]$ or $[\mathbf{1 2}]$.)

COROLlARY 2. (b)-regularity implies 1-regularity.

Proof. The geodesic distance is $d_{A}$ and induces the subspace topology (see [14]).

Proof of the Proposition. It is sufficient to show that $A$ is connected by arcs of finite length. To this purpose it is sufficient to show that for every $X \in \mathscr{S}(A)$ and every $x \in X$ it is possible to join $x$ with a point on the boundary of $X$ by a curve of finite length. This follows in turn from the following fact (whose proof is an exercise in standard calculus): if $\gamma$ is a curve of $\mathbf{R}^{N}$ such that $\{\gamma-\{\gamma(0), \gamma(1)\}, \gamma(0), \gamma(1)\}$ is a Whitney stratification, then $\gamma$ has finite length.

\section{REFERENCES}

[1] S. Alexander, Distance Geometry in Riemannian Manifolds With Boundary, Global diff. geometry and global analysis. Springer Lecture Notes 838 (1981).

[2] R. L. Bishop and R. J. Crittenden, Geometry of Manifolds, Academic Press, New York (1964).

[3] L. M. Blumenthal, Theory and Applications of Distance Geometry, Oxford (1953).

[4] W. M. Boothby, An Introduction to Differentiable Manifolds and Riemannian Geometry, Academic Press, New York (1975). 
[5] H. Busemann, Metric methods in Finsler spaces and in the foundations of geometry, Ann. of Math. Studies, 8, Princeton Univ. Press, Princeton (1942).

[6] - The Geometry of Geodesics, Academic Press, New York (1955).

[7] Recent synthetic differential geometry, Erg. der Math., 54, Springer, Berlin (1970).

[8] A. Ferrari et G. Monti Bragadin, Sur la cohomologie entière des prestratifications abstraites, C.R.A.S. Paris, 279 (1974), 883-886.

[9] J. Mather, Notes on topological stability, Mimeographed notes, Harvard (1970).

[10] H. Natsume, The realization of abstract stratified sets, Kodai Math. J., 3 (1980), $1-7$.

[11] B. L. Reinhart, Foliated manifolds with bundle-like metrics, Ann. Math., 69 (1959), 119-131.

[12] M. Teufel, Abstract prestratified sets are (b)-regular, J. Diff. Geom., 16 (1981), 529-536.

[13] R. Thom, Ensembles et morphismes stratifies, Bull. Amer. Math. Soc., 75 (1969), 240-284.

[14] J. C. Tougeron, Idéaux des fonctions différentiables, Erg. der Math., 71 (1972), Berlin.

[15] A. Verona, Le théorème de deRham pour les prestratifications abstraites, C.R.A.S. Paris, 273 (1971), 886-889.

[16] - Homological properties of abstract prestratifications, Rev. Roum. Math., XVII (1972), 1109-1121.

Received January 24, 1986 and in revised form July 6, 1987.

UNIVERSITÀ di GENOVA

Via L.B. Alberti 4

I-16132 Genova (ITALY) 



\section{PACIFIC JOURNAL OF MATHEMATICS}

\section{EDITORS}

V. S. VARADARAJAN

(Managing Editor)

University of California

Los Angeles, CA 90024

Herbert Clemens

University of Utah

Salt Lake City, UT 84112

R. FINN

Stanford University

Stanford, CA 94305
HERMANN FLASCHKA

University of Arizona

Tucson, AZ 85721

RAMESh A. GANGOLLI

University of Washington Seattle, WA 98195

VAUGHAN F. R. JONES

University of California

Berkeley, CA 94720
ROBION KIRBY

University of California

Berkeley, CA 94720

C. C. MOORE

University of California

Berkeley, CA 94720

HAROLD STARK

University of California, San Diego

La Jolla, CA 92093

\section{ASSOCIATE EDITORS}
R. AREnS
E. F. BECKENBACH
B. H. NEUMANN
F. WOLF
K. YOSHIDA (1906-1982)

\section{SUPPORTING INSTITUTIONS}

UNIVERSITY OF ARIZONA

UNIVERSITY OF OREGON

UNIVERSITY OF BRITISH COLUMBIA UNIVERSITY OF SOUTHERN CALIFORNIA

CALIFORNIA INSTITUTE OF TECHNOLOGY

UNIVERSITY OF CALIFORNIA

STANFORD UNIVERSITY

MONTANA STATE UNIVERSITY

UNIVERSITY OF HAWAII

UNIVERSITY OF NEVADA, RENO

UNIVERSITY OF TOKYO

NEW MEXICO STATE UNIVERSITY

UNIVERSITY OF UTAH

OREGON STATE UNIVERSITY

WASHINGTON STATE UNIVERSITY

UNIVERSITY OF WASHINGTON 


\section{Pacific Journal of Mathematics}

\section{Vol. 133, No. $1 \quad$ March, 1988}

John Anderson, Finitely generated algebras and algebras of solutions to partial differential equations $\ldots \ldots \ldots \ldots \ldots \ldots \ldots \ldots \ldots \ldots \ldots \ldots \ldots$

Junichi Aramaki, On an extension of the Ikehara Tauberian theorem . . . . . 13

Giacomo Monti Bragadin, Abstract Riemannian stratifications .......... 31

Lawrence James Brenton and Richard Hill, On the Diophantine equation

$1=\sum 1 / n_{i}+1 / \prod n_{i}$ and a class of homologically trivial complex

surface singularities .................................41

C. Bruce Hughes, Controlled homotopy topological structures $\ldots . \ldots \ldots \ldots 69$

Peter Wilcox Jones and Takafumi Murai, Positive analytic capacity but zero Buffon needle probability . ...........................999

Gary M. Lieberman, Hölder continuity of the gradient at a corner for the

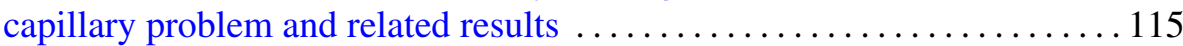

Feng Luo, Representing homology classes of $C \mathbf{P}^{2} \overline{C \mathbf{P}^{2}}$

Claudio Nebbia, Groups of isometries of a tree and the Kunze-Stein

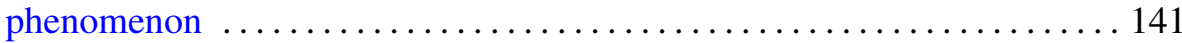

Stefan Richter, Unitary equivalence of invariant subspaces of Bergman and

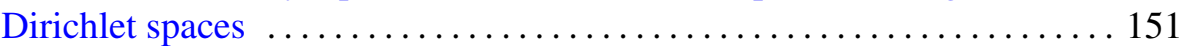

Paul Frederick Ringseth, The Selberg trace formula for groups without Eisenstein series ..................................... 157

Abderrazzak Sersouri, The Mazur property for compact sets $\ldots \ldots \ldots \ldots 185$ Alladi Sitaram, On an analogue of the Wiener Tauberian theorem for symmetric spaces of the noncompact type 